\title{
Comment on: clinical study on surgical treatment of granulomatous lobular mastitis
}

\author{
Sami Akbulut , Tevfik Tolga Sahin^ \\ Department of Surgery and Liver Transplant Institute, Inonu University Faculty of Medicine, Malatya, Turkey \\ Correspondence to: Sami Akbulut, Assoc Prof., FACS. Department of Surgery and Liver Transplant Institute, Inonu University Faculty of Medicine, \\ Elazig Yolu 10. Km, Malatya 44280, Turkey. Email: akbulutsami@gmail.com. \\ Provenance and Peer Review: This article was a free submission to the editorial office, Gland Surgery. The article did not undergo external peer review. \\ Response to: Zhang C, Lei S, Kong C, et al. Clinical study on surgical treatment of granulomatous lobular mastitis. Gland Surg 2019;8:712-22.
}

Submitted May 12, 2020. Accepted for publication Jun 05, 2020.

doi: $10.21037 /$ gs-20-492

View this article at: http://dx.doi.org/10.21037/gs-20-492

We read with great interest the recent article entitled "Clinical study on surgical treatment of granulomatous lobular mastitis" published by Zhang and colleagues (1). The authors stated that they aimed to assess the etiology and features of idiopathic granulomatous mastitis (IGM), as well as the effects of surgery for IGM.

In the introduction section, the authors state that IGM is characterized by lobular necrosis and non-caseating granulomatous inflammation which we completely disagree with. IGM is characterized by non-necrotizing (=noncaseating) granulomatous inflammation. Besides, necrotizing granulomatous inflammation that the authors have stated is the characteristic morphologic appearance of diseases such as tuberculosis (TB), brucellosis, nocardiosis, and Wegener's granulomatosis. Authors have also stated that anti-TB treatment was also included in the management of IGM which we do not agree with also. The anti-TB treatment has no place in the management of IGM; however, TB should be considered in the differential diagnosis of IGM. In current literature, it is seen that many researchers have a controversial approach to the management and diagnosis of IGM. In practice, it is a common fact that many researchers start anti-TB treatment to patients with persistent and/or recurrent IGM because they believe the underlying cause to be TB mastitis. Response to anti-TB therapy does not necessitate anti-TB therapy to be a part of the management of IGM, but it shows that some patients with TB mastitis were misdiagnosed as IGM.
It is clear that the authors do not have a standard algorithm for the diagnosis and management in patients with the suspicion of IGM and this is a common problem in almost all of the studies in the literature. The absence of a specific algorithm and lack of common language is the main source for heterogeneity among the studies in the literature. For example, the authors see the nipple retraction as a risk factor for IGM; however, in our experience, this is a complication of granulomatous inflammation. Therefore, authors and most of the researchers have different views regarding IGM all of which are unclear. Nevertheless, regardless of the etiology of the disease, early diagnosis and treatment are the mainstays for the management of IGM.

Another important point is the heterogeneity of the treatment options for IGM. In our opinion, an entity has a lot of unknown points if there are a lot of treatment options. This is the case for IGM. For example, incision + drainage is considered a standard surgical option; however, the authors considered this option as a non-surgical alternative which we disagree with. In our opinion, there should be a staged treatment approach for IGM. In explanation, conservative approach, antibiotics, steroids, immunosuppressive therapy, and radical surgery should be performed according to a protocol. We are trying to develop an algorithm regarding this issue as well.

In the statistical analysis section, the authors state that the correlation between variables was analyzed using Spearman's Rho correlation coefficient to which we object.

^ ORCID: Sami Akbulut, 0000-0002-6864-7711; Tevfik Tolga Sahin, 0000-0002-9132-6115. 
Phi (Ø) or Cramer's V correlation coefficient should be used for analysis when both variables are categorical. Spearman's Rho correlation coefficient is used for continuous variables that are not normally distributed. In Table 1, the authors have classified the place of accommodation of the patients as city and country; however, using universal expressions such as "rural or urban" would have been more appropriate. The present study aimed to evaluate the risk factors for the recurrence of IGM. The basic method to achieve this goal is to perform univariate and multivariate analysis to determine the risk factors for recurrence and also odds ratio $(\mathrm{OR})$ should be calculated to evaluate the extent of influence of these factors on recurrence. The authors have not calculated an OR for the variables which is a significant limitation of the study.

We evaluated the independent variables presented by the authors in Table 1 and compared them in terms of recurrence in SPSS version 25.0 and Medcalc 19.2.1 software. We present the summary of the results of our calculations in Table 1. According to our results, the risk of recurrence in patients without surgical therapy was 7.8 times higher than the patients who received surgical therapy $(\mathrm{P}=0.008, \mathrm{OR}=7.8)$. Also, we have analyzed the four predisposing factors (including the unknown cause of IGM) that the author has stated and we have found that the presence of unknown causes of IGM had 8.84 times lower than the presence of the other three predisposing factors $(\mathrm{P}=0.031, \mathrm{OR}=8.84)$. Furthermore, three breast

Table 1 Calculation of factors affecting IGM recurrence using univariate analysis

\begin{tabular}{|c|c|c|c|c|c|}
\hline Variable & \multicolumn{2}{|c|}{ IGM recurrence } & \multicolumn{3}{|c|}{ Univariate analysis } \\
\hline Residence & & & 0.108 & 3.07 & $0.93-10.1$ \\
\hline Country & 7 (11.9) & $52(88.1)$ & & & \\
\hline City* & $5(4.2)$ & $114(95.8)$ & & & \\
\hline Married & $12(8.1)$ & 137 (91.9) & & & \\
\hline Unmarried* & $0(0.0)$ & 29 (100.0) & & & \\
\hline Age category I & & & 0.769 & 1.16 & $0.35-3.83$ \\
\hline Other years & $5(7.4)$ & 63 (392.6) & & & \\
\hline 30-45 years & $5(8.6)$ & 53 (91.4) & & & \\
\hline Other years* & $7(5.8)$ & $113(94.2)$ & & & \\
\hline Age category III & & & 1.000 & $* \star 1.68$ & $0.09-30.3$ \\
\hline Other years & $12(7.1)$ & $156(92.9)$ & & & \\
\hline$>45^{\star}$ & $0(0.0)$ & $10(100.0)$ & & & \\
\hline Therapy & & & 0.008 & 7.80 & $2.0-30.4$ \\
\hline Non-surgery & $4(28.6)$ & $10(71.4)$ & & & \\
\hline Surgery* & $8(4.9)$ & $156(95.1)$ & & & \\
\hline
\end{tabular}

Table 1 (continued) 
Table 1 (continued)

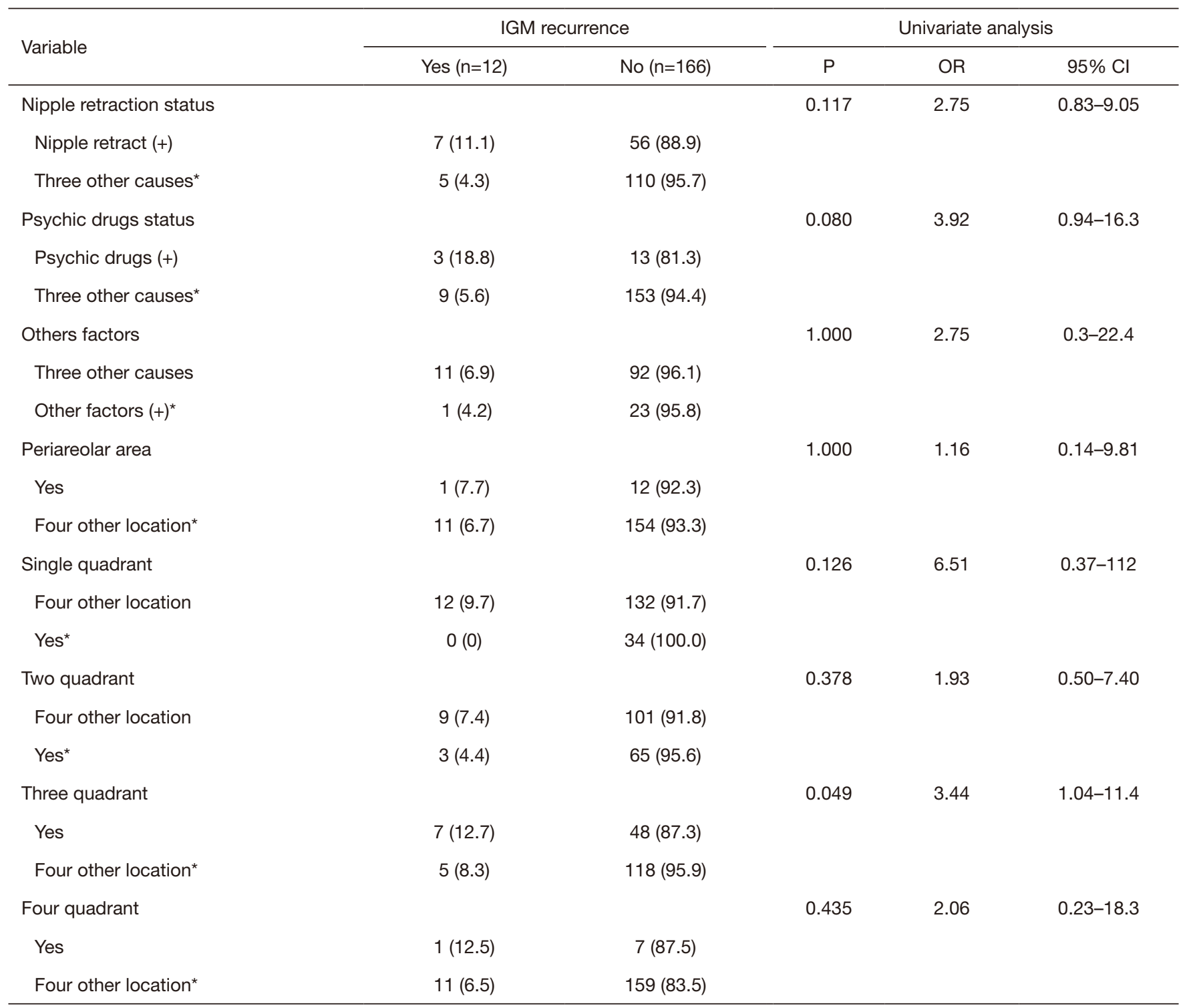

*, reference group; **, Haldane-Anscombe correction performed for statistical analysis, Lesion location: periareolar area, single quadrant, two quadrant, three quadrant, more than three quadrant. IGM, idiopathic granulomatous mastitis.

quadrant involvement by IGM had 3.44 times higher risk when compared to the involvement of other quadrants $(\mathrm{P}=0.049, \mathrm{OR}=3.44)$. However, when all the Phi correlation coefficient are evaluated detail, it is seen that none of these had a moderate to a strong influence on the recurrence risk. This is why multivariate analysis should be performed to all potential candidate variables and the influence of each risk factor should be evaluated. However, the authors have not made any analyses directed against this goal.

\section{Acknowledgments}

Funding: None.

\section{Footnote}

Conflicts of Interest: Both authors have completed the ICMJE uniform disclosure form (available at http://dx.doi. org/10.21037/gs-20-492). The authors have no conflicts of interest to declare. 
Ethical Statement: The authors are accountable for all aspects of the work in ensuring that questions related to the accuracy or integrity of any part of the work are appropriately investigated and resolved.

Open Access Statement: This is an Open Access article distributed in accordance with the Creative Commons Attribution-NonCommercial-NoDerivs 4.0 International License (CC BY-NC-ND 4.0), which permits the noncommercial replication and distribution of the article with

Cite this article as: Akbulut S, Sahin TT. Comment on: clinical study on surgical treatment of granulomatous lobular mastitis. Gland Surg 2020;9(4):1065-1068. doi: 10.21037/gs-20-492 the strict proviso that no changes or edits are made and the original work is properly cited (including links to both the formal publication through the relevant DOI and the license). See: https://creativecommons.org/licenses/by-nc-nd/4.0/.

\section{References}

1. Zhang C, Lei S, Kong C, et al. Clinical study on surgical treatment of granulomatous lobular mastitis. Gland Surg 2019;8:712-22. 